\title{
Les effets indirects de la COVID-19 sur la santé des enfants et des jeunes
}

\author{
Neil Chanchlani MBChB MSc, Francine Buchanan MBSI, Peter J. Gill MD DPhil
}

Citation : CMAJ 2020 August 10;192:E921-7. doi : 10.1503/cmaj.201008-f; diffusion hâtive le 25 juin 2020

Voir la version anglaise de l'article ici : www.cmaj.ca/lookup/doi/10.1503/cmaj.201008

$\mathbf{E}$

n date du 21 juin, 6982 personnes de 19 ans et moins, désignées plus bas comme " les enfants et les jeunes ", avaient reçu un résultat positif au test de dépistage du coronavirus du syndrome respiratoire aigu sévère 2 (SRASCoV-2) au Canada. Parmi ces cas, on a recensé 98 hospitalisations et 20 admissions aux soins intensifs, mais aucun décès ${ }^{1}$. À l'exception des cas de syndrome inflammatoire multisystémique de l'enfant ayant un lien temporel avec le SRASCoV-2, qui est encore mal caractérisé pour le moment ${ }^{2}$, partout dans le monde les enfants et les jeunes sont moins durement touchés par la maladie à coronavirus 2019 (COVID19) que les adultes ${ }^{3}$.

Les pays qui ont vu leurs services de santé grandement bouleversés et qui ont dû imposer des mesures de santé publique à toute la population à cause de la COVID-19 devront probablement composer avec des effets indirects, immédiats et à long terme, de la pandémie sur la santé. Les effets néfastes que peut avoir la maladie sur la santé des enfants et des jeunes sont possiblement sous-estimés. Dans cet article, nous examinons comment la limitation de l'accès aux services de santé de première et de deuxième ligne, la crainte des parents de consulter, la fermeture des garderies et des écoles, l'instabilité financière et d'emploi, et le risque accru d'expériences négatives pour les enfants peuvent faire augmenter la morbidité et la mortalité. Nous traitons de différentes stratégies d'atténuation envisageables, dont la réorganisation des modes de prestation de soins aux enfants et aux jeunes.

\section{Quels effets indirects la pandémie de COVID-19 peut-elle avoir sur la santé physique des enfants et des jeunes?}

Les interruptions de service, les avertissements et les mesures de santé publique en lien avec la pandémie ont considérablement fait diminuer l'utilisation des services de santé par les enfants, les jeunes et les familles. Au Royaume-Uni ${ }^{4}$, en Irlande ${ }^{5}$

\section{POINTS CLÉS}

- Peu d'enfants et de jeunes ont souffert de la maladie à coronavirus 2019 (COVID-19) ou ont été hospitalisés pour une raison directement liée à cette maladie. Cependant, la pandémie a fait fermer les garderies et les écoles et causé des interruptions de service qui ont limité l'accès aux services de santé de première et de deuxième ligne, fait craindre aux parents de consulter et engendré une instabilité financière et d'emploi.

- On s'attend à ce que les expériences négatives de l'enfance, comme la violence familiale, les traumatismes non accidentels et les troubles mentaux, augmentent pendant le confinement et s'aggravent pendant la récession économique prévue.

- Il faut continuer de prodiguer des soins communautaires et hospitaliers en toute sécurité, virtuellement ou, pour certains groupes, en personne.

- Il faut aussi répondre de manière claire et transparente aux questions des enfants, des jeunes et des familles sur la continuité des soins et, le cas échéant, la réorganisation des services.

- Les décisions concernant la reprise de la vie sociale, le retour à la normale des services de santé et les changements dans les services devraient être prises à partir de données recueillies de façon systématique et des expériences vécues par les familles.

et en Italie ${ }^{6}$, les données d'admission recueillies d'office montrent que pendant le confinement, le nombre d'enfants vus à l'urgence et le nombre d'enfants hospitalisés ont pu connaître une baisse atteignant $75 \%$ par rapport à la même période des années précédentes. L'Hôpital SickKids de Toronto, lui, a noté une réduction des visites à l'urgence de $30 \%$ en mars et de $62 \%$ en avril 2020, comparativement à 2019 (Dr Karim Jessa, Hôpital SickKids, Toronto (Ontario) : communication personnelle, 2020).

Certains parents ont retardé la visite à l'hôpital de leur enfant, rapportent plusieurs sources. En 5 jours, 5 hôpitaux 
italiens ont vu 12 cas de visite tardive pour des maladies autres que la COVID-19: 6 ont nécessité des soins intensifs, et 4 se sont soldés par un décès ${ }^{6}$. Il semble qu'on observe la même tendance dans les pays à revenu faible ou intermédiaire. Dans un hôpital pédiatrique indien de troisième ligne, par exemple, le nombre d'enfants atteints d'une acidocétose diabétique grave se présentant à l'hôpital était plus élevé en avril 2020, en plein confinement, que l'année précédente ${ }^{7}$. Pourquoi ne pas avoir cherché à obtenir des soins plus rapidement? Il y a différentes causes possibles: la peur des parents de s'exposer au SRAS-CoV-2 à l'hôpital ou dans les transports en commun, l'absence de gardien pour les autres enfants (surtout en cas de monoparentalité), l'impossibilité de consulter en première ligne en raison d'une interruption de service, et les modifications aux politiques de visite des hôpitaux qui obligeraient les parents à y laisser leur enfant seul.

D'un autre côté, la forte réduction des interactions sociales et des occasions de jeu des enfants et des jeunes due aux politiques nationales de confinement - dont l'imposition d'une quarantaine et de restrictions des déplacements - a probablement freiné la transmission d'autres micro-organismes répandus et limité l'exposition à des déclencheurs de problèmes respiratoires courants et à des allergènes, et possiblement réduit le nombre de traumatismes accidentels.

Avec la fermeture d'établissements de première ligne motivée par l'incapacité à appliquer des pratiques optimales de contrôle des infections, combinée à la crainte des parents de se rendre en milieu de soins, beaucoup d'enfants n'ont pas reçu leurs vaccins ou les recevront plus tard, ce qui menace l'immunité collective et risque d'engendrer des éclosions de maladies infectieuses évitables. Selon un sondage récemment mené auprès de 1000 pédiatres aux États-Unis, le taux de vaccination contre la rougeole, les oreillons et la rubéole était $50 \%$ plus bas dans la semaine du 5 avril 2020 que 2 mois auparavant, et $42 \%$ plus bas pour la diphtérie et la coqueluche ${ }^{8}$. Dernièrement, le programme américain Vaccines For Children a fait état d'une forte chute du taux de vaccination des enfants contre des maladies autres que la grippe, dont la rougeole, les oreillons et la rubéole, entre 2019 et la période du 6 janvier au 19 avril 2020 (figure 1$)^{9}$.

Le 22 mai, l'Organisation mondiale de la Santé a annoncé que 68 pays à faible revenu avaient suspendu ou reporté la vaccination systématique des enfants, une mesure qui touche plus de 80 millions de bébés de moins d'un an et qui s'explique en partie par les difficultés dans le transport international des doses et une pénurie de professionnels de la santé ${ }^{10}$. Cette décision fera diminuer l'immunité collective contre les maladies infectieuses infantiles à l'échelle mondiale.

Une autre source potentielle d'effets indésirables est l'absence de bilans de santé réguliers. Prenons l'exemple des évaluations développementales : il est particulièrement inquiétant de penser qu'elles n'ont pas lieu, compte tenu qu'environ $15 \%$ des enfants ont un retard dans au moins 1 domaine de développement et qu'on sait que le dépistage précoce améliore le développement cognitif et la santé ${ }^{11}$.

\section{Quels effets peuvent subir les enfants, les jeunes et les familles sur les plans social et psychologique?}

Les expériences négatives de l'enfance, comme la maltraitance, la pauvreté et l'insécurité alimentaire, sont associées à des problèmes de santé mentale, à l'obésité et à des maladies cardiovasculaires à l'âge adulte ${ }^{12}$. Elles seraient plus courantes chez les enfants et les jeunes en situation d'isolement social obligatoire, surtout les nouveaux réfugiés, les membres de familles marginalisées et les Autochtones, déjà aux prises avec de mauvaises conditions de logement, des difficultés financières et l'insécurité alimentaire ${ }^{13}$.

Les restrictions et les annulations de visites des familles à risque par les services de protection de la jeunesse, et la possibilité que les visites supervisées des parents biologiques (par exemple de mères qui allaitent) aux enfants en famille d'accueil se fassent plus rares, posent aussi des risques. Maintenant que la récession économique s'accentue, on s'attend à une hausse de la violence familiale ${ }^{14}$, accompagnée d'une recrudescence des blessures non accidentelles ${ }^{15}$ et des traumatismes psychologiques chez les enfants et les jeunes ${ }^{16}$. Une aggravation des iniquités économiques et en santé est aussi à prévoir.

En mars, l'Organisation des Nations Unies pour l'éducation, la science et la culture (UNESCO) a rapporté que plus de 1,19 milliard d'élèves de 150 pays n'allaient plus à l'école en raison d'un confinement décrété par leur gouvernement. La fermeture des écoles exacerbe l'insécurité alimentaire pour les nombreux enfants qui dépendent des repas qui y sont fournis, et enlève un lieu sûr aux enfants vulnérables et placés. De plus, elle bouleverse le quotidien des familles : les parents peinent à pourvoir aux besoins éducatifs de leurs enfants alors qu'ils doivent conjuguer tâches professionnelles et rôle parental ou faire face à une incertitude financière. D'après un sondage mené auprès de 245 mères italiennes d'enfants de 2 à 5 ans, le confinement national a déréglé les horaires de sommeil, causé de l'ennui et fait augmenter les problèmes affectifs et comportementaux en affaiblissant la maîtrise de soi, chez les mères comme chez les enfants ${ }^{17}$.

En période d'éloignement physique et de confinement, les enfants et les jeunes n'interagissent plus entre eux, n'ont peutêtre plus de routine établie et sont vraisemblablement moins actifs physiquement. En effet, une enquête sur les habitudes de vie effectuée avant et après l'implantation de mesures de santé publique liées à la COVID-19 montre que chez les 2426 enfants et adolescents (6-17 ans) sondés, qui fréquentaient 5 écoles de Shanghai, en Chine, le nombre d'heures d'activité physique par semaine a diminué de 7,3, tandis que le temps d'écran a augmenté de 30 heures par semaine ${ }^{18}$. Lorsqu'il est prolongé, le temps d'écran peut accroître la difficulté à se concentrer, la tristesse et l'irritabilité, comme l'ont décrit des adolescents chinois, et ainsi déclencher des premiers signes de dépression et d'anxiété ${ }^{19}$.

Les consignes d'éloignement physique et de confinement ont des implications encore plus grandes pour les enfants ayant besoin de soins de santé, comme des services de soutien pour 
des problèmes de développement, qui peuvent être interrompus ou réduits, ou comme l'oxygénothérapie à domicile, qui pour des questions d'approvisionnement pourrait devenir impossible. De surcroît, les familles d'enfants qui ont des besoins médicaux particuliers risquent de devoir prendre une décision difficile, entre continuer de laisser entrer dans leur domicile des travailleurs en services communautaires qui les aident pour la prestation de soins essentiels - souvent avec de l'équipement de protection individuelle inadéquat - et s'occuper seules de l'enfant et renoncer à cette possibilité de répit.

Au Canada, les politiques d'augmentation de salaire des travailleurs essentiels en santé, dont les infirmières et les préposés aux bénéficiaires, pourraient contraindre certaines familles à gratter les fonds de tiroirs pour se permettre les services dont elles ont besoin, puisqu'il n'y a pas eu de hausse dans les allocations gouvernementales qui leur sont destinées, comme le Programme de services particuliers à domicile et le Financement accru des services de relève pour les enfants dont la santé est fragile ou dépend de moyens technologiques de l'Ontario ${ }^{20}$. En Italie, selon des soignants, des familles forcées de prendre soin d'un enfant ou d'un adolescent ayant un trouble neurodéveloppemental à domicile sans aide ont vécu des difficultés ${ }^{21}$, et les comportements violents, les comportements d'automutilation non suicidaire et les tentatives de suicide ont augmenté chez les jeunes ${ }^{22}$.

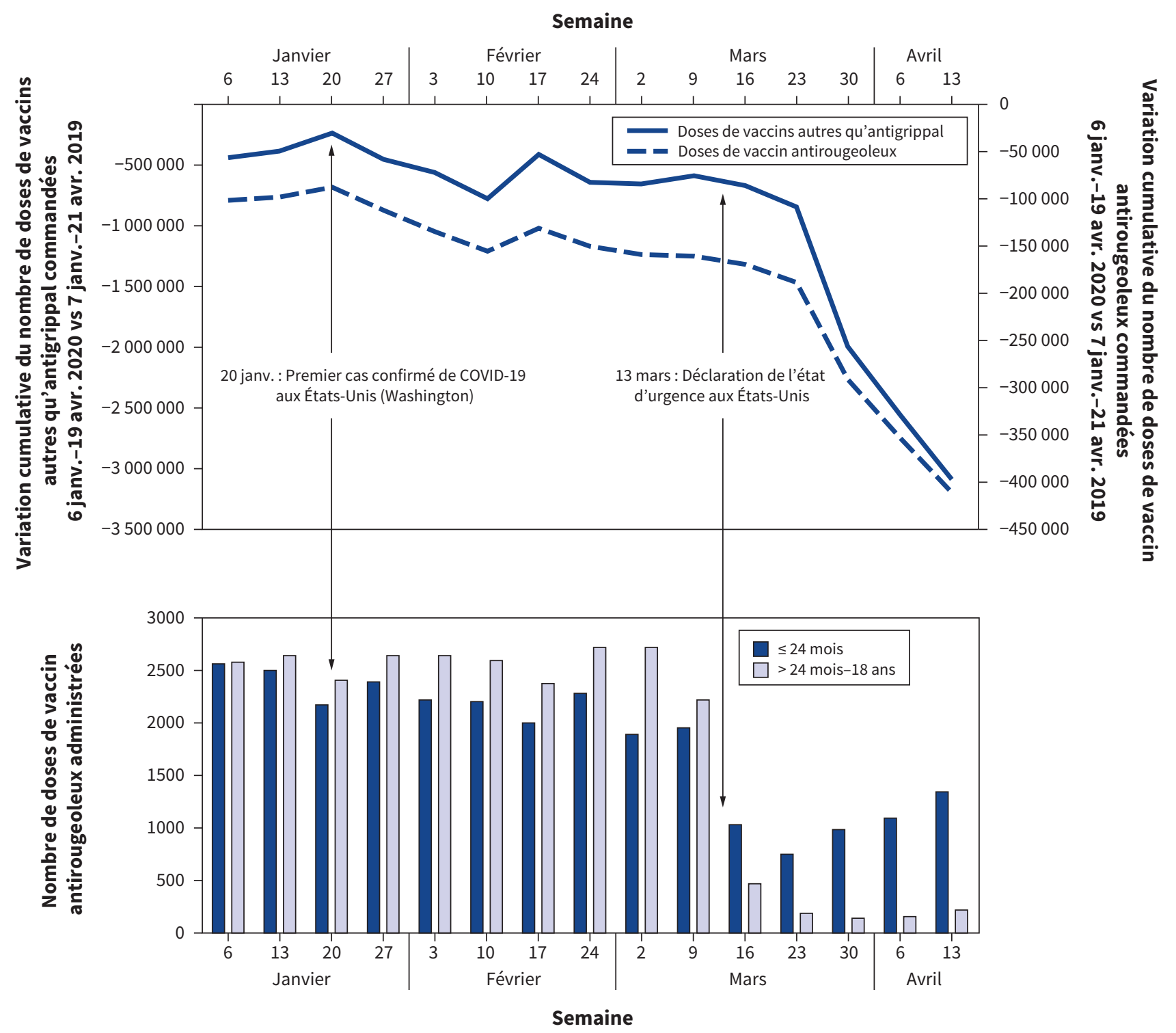

Figure 1 : Différence, par semaine, dans les commandes de doses pour le programme Vaccines For Children* et le nombre de doses administrées dans le cadre du Vaccine Safety Datalink†, pour les vaccins du calendrier d'immunisation des enfants - États-Unis, 6 janvier-19 avril 2020․ * Les données du programme Vaccines For Children montrent la différence cumulative hebdomadaire dans le nombre de doses de vaccins autres qu'antigrippal et de vaccin antirougeoleux payées par le programme qui ont été commandées par les professionnels de la santé, entre la période du 7 janvier-21 avril 2019 et la période du 6 janvier-19 avril 2020. †Les données du Vaccine Safety Datalink indiquent le nombre de doses de vaccin antirougeoleux administrées en une semaine, selon le groupe d'âge ( $\leq 24$ mois et $>24$ mois-18 ans). Remarque : COVID-19 = maladie à coronavirus 2019. 


\section{Comment soigner les enfants, les jeunes et les familles en toute sécurité pendant la pandémie?}

\section{Systèmes de santé réactifs et adaptatifs}

Les médecins de famille et les pédiatres doivent dire aux parents et aux travailleurs concernés, dont les éducatrices en garderie, les enseignants et les travailleurs sociaux, que les services de santé sont ouverts et accessibles si un enfant ou un jeune en a besoin. Il faut continuer de prodiguer des services de santé en toute sécurité. L'encadré $n^{\circ} 1$ énumère les services externes à donner en personne et ceux qui peuvent se faire virtuellement ${ }^{23}$.

Le personnel des services de pédiatrie en milieu hospitalier devrait entamer la discussion avec les enfants, les jeunes et les familles afin de trouver des solutions de rechange pour la prestation de soins spécialisés - comme déplacer les salles de perfusion qui servent à la chimiothérapie ou à l'injection de médicaments biologiques dans des endroits où le risque d'exposition au SRAS-CoV-2 est faible, réorganiser les services de deuxième ligne pour les offrir en services communautaires de proximité (p. ex., pour les patients souffrant de troubles alimentaires) et faire suivre des thérapies cruciales (physiothérapie, ergothérapie, thérapie cognitivocomportementale, etc.) sur des plateformes virtuelles. On assiste déjà à des changements novateurs, notamment la création d'une urgence pour enfants virtuelle dans plusieurs hôpitaux pédiatriques canadiens ${ }^{24}$.

\section{Communication claire avec les enfants et les familles}

Afin de mieux soutenir les familles, il est important de mieux comprendre comment elles prennent des décisions concernant les besoins complexes des enfants et des jeunes quand des mesures de lutte contre la pandémie sont en vigueur, en intégrant à la fois des considérations pratiques et personnelles. Les familles pourraient, avec raison, être réticentes à venir consulter en personne, ce qui demanderait qu'on leur explique avec soin la raison pour laquelle leur présence est requise. L'application d'approches pratiques est nécessaire pour les familles de jeunes enfants et les enfants ayant besoin de soins médicaux dont la famille n'est pas en mesure de respecter des directives strictes.

Les professionnels de la santé devraient être capables de justifier chacune de leurs recommandations et chacun des changements apportés aux services de première et de deuxième ligne, pour donner aux familles la possibilité d'évaluer si tous les facteurs qu'elles jugent importants sont pris en compte. Le tableau 1 énonce des questions courantes que les cliniciens pourraient se faire poser et propose des stratégies de communication pour leur répondre. Ici, la clé est l'anticipation des préoccupations.

Il faudrait, dans le but d'atténuer les effets des mesures de santé publique sur la santé mentale des enfants, publier des messages et des documents de santé publique qui parlent du risque d'une façon adaptée à l'âge. Il existe maintenant plusieurs ressources pour aider les parents à bien jouer leur rôle
Encadré $n^{\circ} 1$ : Services externes prioritaires pour les enfants et les jeunes pendant la pandémie de COVID-19

\section{En personne}

- Vaccination, surtout pour les populations à risque

- Soins du nouveau-né, y compris la pesée, le dépistage de l'ictère et l'évaluation de l'allaitement

- Soupçons de retard staturo-pondéral

- Blessure légère, pour éviter une visite à l'urgence

- Maladie aiguë

- Soupçons de maltraitance

- Programmes de dépistage (p. ex., des problèmes auditifs)

- Administration de médicaments essentiels (p. ex., chimiothérapie)

\section{En ligne}

- Surveillance du développement (p. ex., suivi à 18 mois)

- Problèmes à l'école, d'apprentissage ou de comportement

- Prise en charge d'une maladie chronique (p. ex., diabète de type 1, épilepsie, asthme)

- Renouvellement d'ordonnances

- Soupçons naissants ou accrus concernant la santé mentale

Remarque : COVID-19 = maladie à coronavirus 2019

durant la pandémie, et les soignants devraient être encouragés à faire découvrir ce genre d'outils aux familles, aux enfants et aux jeunes (figure 2 et encadré $n^{\circ} 2$ ).

\section{Prise de décisions fondée sur les données}

Les données sont essentielles pour arriver à endiguer la pandémie, et les enfants et les jeunes ne doivent pas être laissés de côté. Nous devons améliorer notre connaissance des relations multidirectionnelles entre la santé des enfants, le gouvernement, les mesures de santé publique contre la pandémie et les réactions de la société à la crise. Les patients et les familles devraient participer à la définition des objectifs de production de données et des critères d'évaluation pertinents en lien avec la prestation de soins de santé, l'éducation et les services sociaux.

Les politiques concernant le retour à l'école et les activités sociales doivent prendre en compte les effets sur la santé physique et mentale des enfants et des jeunes en regard de la transmissibilité du virus ${ }^{25}$, et être communiquées de manière à répondre aux préoccupations des familles. En ce moment, les politiques varient au pays: dans certaines provinces, les écoles ont rouvert partiellement ou totalement, alors que dans d'autres, elles sont restées fermées pour le reste de l'année scolaire. Les activités sociales destinées aux enfants et aux jeunes durant les vacances d'été, comme les camps de loisirs, devraient faire l'objet d'une collecte de données détaillées, à l'interne, qui indiqueraient si les participants contractent et transmettent le SRAS-CoV-2 sur place, et, le cas 
Comment le personnel de la clinique ou de l'hôpital va-t-il garder mon enfant en sécurité?

Que se passera-t-il si je veux consulter?

Pourquoi dois-je me présenter en personne? Pourquoi je n'ai pas une consultation virtuelle?

Comment me rendre à la clinique ou à l'hôpital en toute sécurité?

Comment avez-vous pris cette décision pour mon enfant?

À qui m'adresser si j’ai des questions?

Quoi faire pour protéger ma famille?

Que faire si une personne chez moi contracte la COVID-19?
Anticiper les préoccupations de la famille, et indiquer comment les soins de première ou de deuxième ligne ont été réorganisés, par exemple

- Réaménager l'espace pour permettre l'éloignement physique : placer les chaises à bonne distance les unes des autres, prévoir plusieurs espaces de jeu pour les enfants, etc.

- Indiquer la fréquence de nettoyage des salles, des chaises et des jouets

- Avoir de l'équipement de protection individuelle pour le personnel et les patients

Se tenir au courant des politiques et des procédures de l'établissement et parler de ce qu'elles impliquent pour les patients:

- Nombre de parents accompagnateurs autorisés

- Possibilité de rester avec l'enfant à l'hôpital

- Réactions possibles des enfants et des jeunes au port d'équipement de protection individuelle Donner la raison pour laquelle la présence en personne est requise (p. ex., examen physique)

Demander au patient les plages horaires qui lui conviennent, d'autant plus que les services de transports en commun sont réduits

Offrir le stationnement gratuit ou à rabais, si possible

Citer les services de navette fiables

Le cas échéant, expliquer les processus utilisés à cause de la COVID-19 et discuter des risques et des avantages de façon ouverte et transparente

Donner votre numéro de téléphone ou votre adresse courriel, pour éviter que les familles aillent consulter en personne

Suggérer des stratégies, par exemple :

- Nommer des activités récréatives sécuritaires

- Donner des conseils pratiques aux familles de jeunes enfants et aux familles qui comptent un enfant ayant besoin de soins médicaux et qui ne sont pas capables de suivre des règles strictes

- Donner une liste à jour des services de soutien locaux

Suggérer des stratégies en fonction des contraintes de la famille, telles que :

- La possibilité de s'isoler en toute sécurité à l'intérieur du domicile

- L'accès à un gardien qui sait comment répondre aux besoins particuliers de l'enfant

- La possibilité d'obtenir de la nourriture, des fournitures et des médicaments sans sortir de la maison

Remarque : COVID-19 = maladie à coronavirus 2019.

échéant, quels sont les effets sur les membres de leur foyer et de leur communauté.

Des sondages bien conçus, distribués de façon proactive, aideraient à saisir l'expérience des enfants, des jeunes et des familles en évaluant entre autres l'insécurité alimentaire et de logement et les difficultés financières familiales, la santé mentale des parents, et la présence de conflits et de violence dans la relation entre les parents. L'estimation rigoureuse de l'ampleur des problèmes économiques attribuables aux mesures de santé publique devrait guider le gouvernement dans ses interventions, par exemple lui indiquer s'il convient, pour prévenir les expériences négatives de l'enfance, d'assurer un revenu de base universel, de transférer de l'argent sans conditions aux familles à faible revenu, ou d'augmenter le financement des programmes pour personnes handicapées ${ }^{26}$.

L'accès à des données nationales en temps réel sur les visites à l'urgence et les hospitalisations et l'existence de définitions de cas claires du report de soins favoriseraient la détection hâtive des situations de report (hausse du nombre d'enfants faisant des crises d'asthme aiguë, augmentation de la fréquence des crises d'épilepsie, etc.) et des séquelles de la COVID-19 (comme le syndrome inflammatoire multisystémique de l'enfant ayant un lien temporel avec le SRAS-CoV-2). Ces données permettraient la comparaison des critères d'évaluation selon le moment et l'endroit, et aideraient les chercheurs et les responsables des politiques à déterminer les effets des mesures, à faire des plans pour la suite de la pandémie et à se préparer pour les prochaines.

Des initiatives locales sont en cours, mais idéalement, le Canada se doterait d'un système de surveillance qui répertorierait les issues négatives liées à une consultation tardive signalées par les médecins, comme l'ont fait le Royaume-Uni (www.rcpch.ac.uk/key-topics/covid-19) et la Nouvelle-Zélande (www.otago.ac.nz/nzpsu/current -studies/\#covid-19) avec l'appui des associations nationales de pédiatres. 

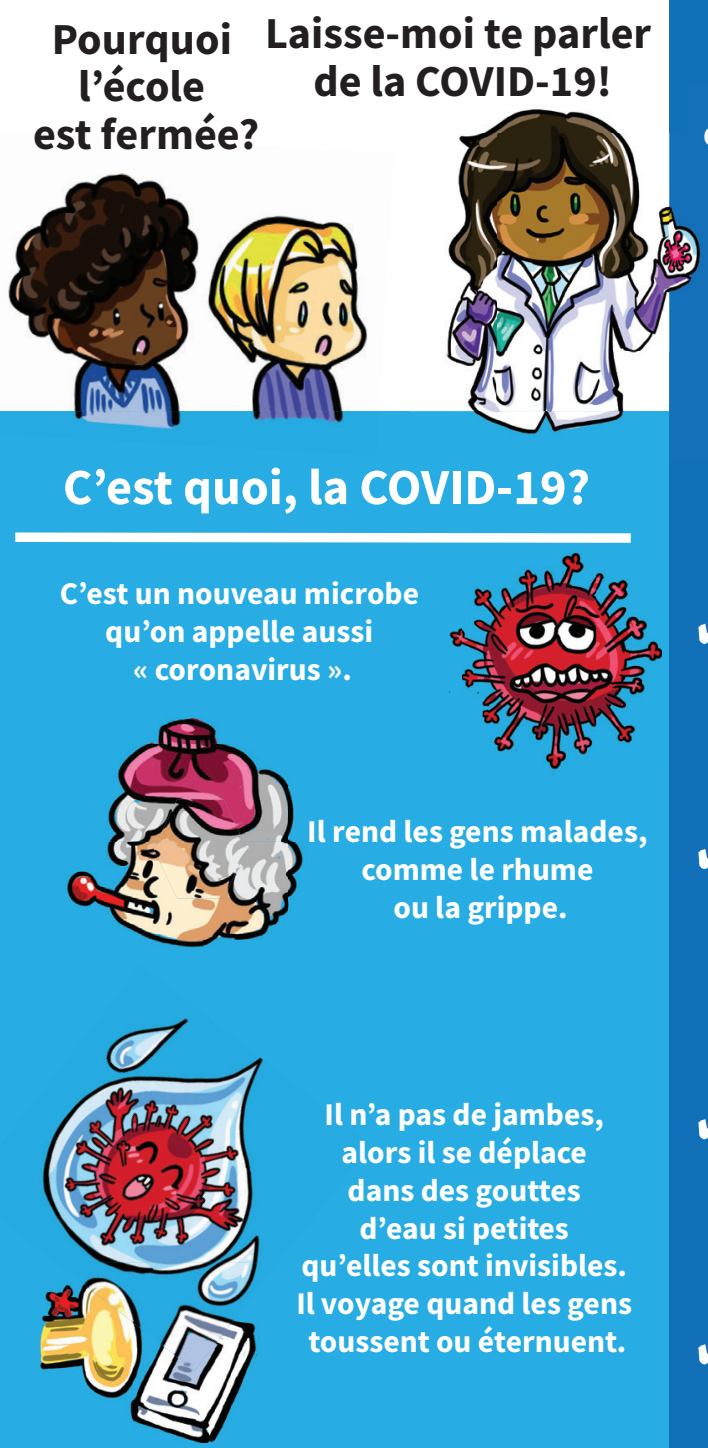

Il se tient aussi sur les objets comme les poignées de porte et les téléphones.

Il n'aime pas le savon, et disparaît quand tu te laves les mains!

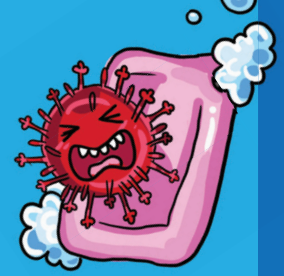

Beaucoup de médecins et

de scientifiques cherchent

des médicaments qui empêcheraient

la COVID-19 de rendre

les gens malades, mais pour l'instant...

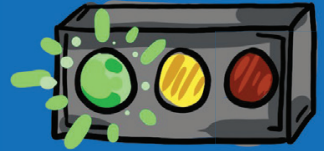

\section{Bloque les microbes!}

Lave-toi les mains avant de manger, après t'être touché le visage et après être allé à la toilette.

Pendant que tu frottes, chante " Bonne fête » ou "Frère Jacques »!

Mets-toi le coude devant la bouche quand tu tousses ou éternues.

Aide ta famille en faisant une tâche domestique chaque jour!

Pose tes questions à un adulte de confiance, et parle-lui de tes peurs. C'est un sentiment normal! Les applis de méditation, l'exercice et la lecture peuvent t'aider à te sentir mieux.

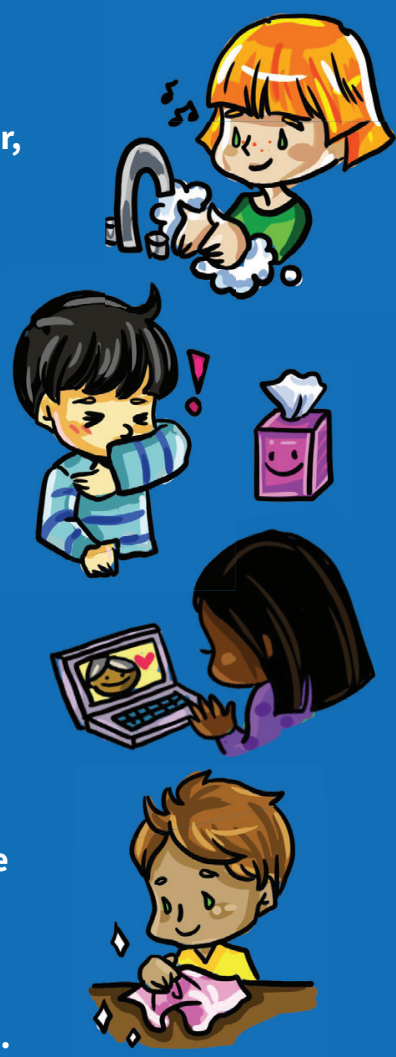

- Ne te touche pas le visage ni les yeux.

- Ne fais pas de câlins ou de bisous à tes grands-parents.

- Si tu te sens malade, ne t'inquiète pas; dis-le à un adulte de confiance et repose-toi quelques jours pour mieux guérir. 
Encadré $n^{\circ} 2$ : Ressources pour expliquer la pandémie de COVID-19 aux enfants et aux jeunes, destinées aux parents et aux tuteurs

- COVID-19 Health Literacy Project. (Traductions dans de nombreuses langues disponibles) (https://covid19healthliteracyproject.com/\#)

- Société canadienne de pédiatrie. Information et ressources sur la COVID19 pour les pédiatres (https://www.cps.ca/fr/tools-outils/ information-et-ressources-sur-la-covid-19-pour-les-pediatres)

- Soins de nos enfants. La COVID-19 et votre enfant (https://www. soinsdenosenfants.cps.ca/handouts/the-2019-novel-coronavirus-covid-19)

- Centers for Disease Control and Prevention. Parler de la maladie à coronavirus 2019 avec les enfants (www.cdc.gov/coronavirus/2019-ncov/ daily-life-coping/talking-with-children.html) [en anglais]

- UNICEF. Comment assurer la sécurité des enfants en ligne pendant la pandémie de COVID-19: trucs pour les parents et les tuteurs (www. unicef.org/laos/stories/keeping-children-safe-online-during-covid-19pandemic) [en anglais]

- Children's Commissioner (Royaume-Uni). Guide pour enfants sur le coronavirus (www.childrenscommissioner.gov.uk/publication/childrens -guide-to-coronavirus) [en anglais]

- Royal College of Paediatrics and Child Health (Royaume-Uni). COVID-19 Ressources pour les parents et les tuteurs (www.rcpch.ac.uk/resources/ covid-19-resources-parents-carers\#downloadBox) [en anglais]

Remarque : COVID-19 = maladie à coronavirus 2019.

\section{Conclusion}

Même s'il est vrai que les cas graves de COVID-19 semblent rares chez les enfants et les jeunes, il est probable que cette tranche de la population connaisse des effets indirects importants de la maladie sur les plans physique, social et psychologique, associés à la réduction des services non urgents et à l'implantation des mesures générales d'endiguement. Nous avons un devoir envers eux : mesurer les effets indirects de la pandémie sur sa santé et prendre les moyens nécessaires pour limiter les dommages collatéraux.

\section{Références}

1. Coronavirus disease 2019 (COVID-19): epidemiology update. Ottawa: Public Health Agency of Canada; updated 2020 June 15. Accessible ici : https:// health-infobase.canada.ca/covid-19/epidemiological-summary-covid-19 -cases.html (consulté le 21 juin 2020).

2. Riphagen S, Gomez X, Gonzalez-Martinez C, et al. Hyperinflammatory shock in children during COVID-19 pandemic. Lancet 2020;395:1607-8.

3. Dong Y, Mo X, Hu Y, et al. Epidemiology of COVID-19 among children in China. Pediatrics 2020;145:e20200702.

4. Isba R, Edge R, Jenner R, et al. Where have all the children gone? Decreases in paediatric emergency department attendances at the start of the COVID19 pandemic of 2020. Arch Dis Child 2020 May 6 [Cyberpublication avant impression]. doi : 10.1136/archdischild-2020-319385.

5. Dann L, Fitzsimons J, Gorman KM, et al. Disappearing act: COVID-19 and paediatric emergency department attendances. Arch Dis Child 2020 June 9 [Cyberpublication avant impression]. doi : 10.1136/ archdischild-2020-319654.

6. Lazzerini M, Barbi E, Apicella A, et al. Delayed access or provision of care in Italy resulting from fear of COVID-19. Lancet Child Adolesc Health 2020;4:e10-1.
7. Dayal D, Gupta S, Raithatha D, et al. Missing during COVID-19 lockdown: children with new-onset type 1 diabetes. Research Square 2020 May 13 [Cyberpublication avant impression]. doi : 10.21203/rs.3.rs-28594/v1.

8. Hoffman J. Vaccine rates drop dangerously as parents avoid doctor's visits. The New York Times 2020 Apr. 23. Accessible ici : www.nytimes. com/2020/04/23/health/coronavirus-measles-vaccines.html (consulté le 23 avril 2020).

9. Santoli JM, Lindley MC, DeSilva MB, et al. Effects of the COVID-19 pandemic on routine pediatric vaccine ordering and administration - United States, 2020. MMWR Morb Mortal Wkly Rep 2020;69:591-3.

10. At least 80 million children under one at risk of diseases such as diphtheria, measles and polio as COVID-19 disrupts routine vaccination efforts, warn Gavi, WHO and UNICEF [communiqué de presse]. Geneva: World Health Organization; 2020 May 22; 22 mai 2020. Accessible ici : www.who.int/news-room/ detail/22-05-2020-at-least-80-million-children-under-one-at-risk-of-diseases -such-as-diphtheria-measles-and-polio-as-covid-19-disrupts-routine-vaccination -efforts-warn-gavi-who-and-unicef (consulté le 22 mai 2020).

11. Vitrikas K, Savard D, Bucaj M. Developmental delay: when and how to screen. Am Fam Physician 2017;96:36-43.

12. Danese A, Moffitt TE, Harrington $\mathrm{H}$, et al. Adverse childhood experiences and adult risk factors for age-related disease: depression, inflammation, and clustering of metabolic risk markers. Arch Pediatr Adolesc Med 2009;163: 1135-43.

13. Willows N, Veugelers $\mathrm{P}$, Raine $\mathrm{K}$, et al. Associations between household food insecurity and health outcomes in the Aboriginal population (excluding reserves). Health Rep 2011;22:15-20.

14. Wong CA, Ming D, Maslow G, et al. Mitigating the impacts of the COVID-19 pandemic response on at-risk children. Pediatrics 2020 Apr. 21 [Cyberpublication avant impression]. doi : 10.1542/peds.2020-0973.

15. Huang MI, O'Riordan MA, Fitzenrider E, et al. Increased incidence of nonaccidental head trauma in infants associated with the economic recession. $J$ Neurosurg Pediatr 2011;8:171-6

16. Sprang G, Silman M. Posttraumatic stress disorder in parents and youth after health-related disasters. Disaster Med Public Health Prep 2013;7:105-10.

17. Di Giorgio E, Di Riso D, Mioni G, et al. The interplay between mothers' and children behavioral and psychological factors during COVID-19: an Italian study. PsyArXiv 2020 Apr. 30 [Cyberpublication avant impression]. doi : 10.31234/osf. io/dqk7h.

18. Xiang M, Zhang Z, Kuwahara K. Impact of COVID-19 pandemic on children and adolescents' lifestyle behavior larger than expected. Prog Cardiovasc Dis 2020 Apr. 30 [Cyberpublication avant impression]. S0033-0620(20)30096-7. doi : 10.1016/j.pcad.2020.04.013.

19. Xie X, Xue Q, Zhou Y, et al. Mental health status among children in home confinement during the coronavirus disease 2019 outbreak in Hubei Province, China. JAMA Pediatr 2020;e201619 [Cyberpublication avant impression]. doi : 10.1001/jamapediatrics.2020.1619.

20. Ontario Ministry of Children, Community and Social Services. Temporary changes to the Special Services at Home (SSAH) and enhanced respite for medically fragile and/or technology dependent children programs [addendum]. Toronto: Queen's Printer for Ontario; modified 2020 May 21. Accessible ici : www.children.gov.on.ca/htdocs/English/specialneeds/ specialservices/addendum-april2020.aspx (consulté le 16 juin 2020).

21. de Girolamo G, Cerveri G, Clerici M, et al. Mental health in the coronavirus disease 2019 emergency - the Italian response. JAMA Psychiatry 2020 Apr. 30 [Cyberpublication avant impression]. doi : 10.1001/jamapsychiatry.2020.1276.

22. Cognigni M. An Italian paediatric department at the time of coronavirus: a resident's point of view. Arch Dis Child 2020 Apr. 27 [Cyberpublication avant impression]. doi : 10.1136/archdischild-2020-319299.

23. Sharon R. Providing virtual care during a pandemic: a guide to telemedicine in the paediatric office [blog]. Ottawa: Canadian Paediatric Society; 2020 Mar. 26. Accessible ici : www.cps.ca/en/blog-blogue/virtual-care-during-a-pandemic (consulté le 20 avril 2020).

24. Rosenfield D, Levinter J, Mizzi T, et al. Role for isolated emergency medicine physicians during a pandemic. CJEM 2020 May 5 [Cyberpublication avant impression]. doi : 10.1017/cem.2020.390.

25. Viner RM, Russell SJ, Croker H, et al. School closure and management practices during coronavirus outbreaks including COVID-19: a rapid systematic review. Lancet Child Adolesc Health 2020;4:397-404.

26. Brownell MD, Chartier MJ, Nickel NC, et al.; PATHS Equity for Children Team. Unconditional prenatal income supplement and birth outcomes. Pediatrics 2016;137:e20152992. 
Intérêts concurrents : Neil Chanchlani déclare avoir reçu une bourse de recherche clinique de Crohn's and Colitis UK. Aucun autre intérêt concurrent déclaré.

Cet article a été révisé par des pairs.

Affiliations : Royal Devon and Exeter NHS Foundation Trust (Chanchlani); Université d'Exeter (Chanchlani), Exeter, Royaume-Uni; Institut des politiques, de la gestion et de l'évaluation de la santé (Buchanan, Gill), Université de Toronto; Hôpital pour enfants malades (SickKids) de Toronto (Buchanan, Gill); Département de pédiatrie (Gill), Université de Toronto, Toronto, Ontario.

Collaborateurs : Neil Chanchlani et Peter Gill ont contribué à la conceptualisation du projet et ont rédigé la première version de l'article. Tous les auteurs ont participé à la rédaction et à la révision du manuscrit, ont donné leur approbation finale pour la version destinée à être publiée et assument l'entière responsabilité de tous les aspects du travail.
Propriété intellectuelle du contenu : Il s'agit d'un article en libre accès distribué conformément aux modalités de la licence Creative Commons Attribution (CC BY-NC-ND 4.0), qui permet l'utilisation, la diffusion et la reproduction dans tout médium à la condition que la publication originale soit adéquatement citée, que l'utilisation se fasse à des fins non commerciales (c.-à-d., recherche ou éducation) et qu'aucune modification ni adaptation n'y soit apportée. Voir : https://creativecommons.org/ licenses/by-nc-nd/4.0/deed.fr

Remerciements : Les auteurs remercient Anne Fuller pour ses suggestions.

Déclaration d'intérêts : Neil Chanchlani est rédacteur associé pour le CMAJ, mais n'a pas participé au processus ayant mené au choix de cet article.

Correspondance : Neil Chanchlani, nchanchlani@doctors.org.uk 